\title{
ATM With Eye Tracker Password Authentication
}

\author{
Mrs. Anu D ${ }^{1}$, Harini L ${ }^{2}$, Gayathri $\mathbf{H}^{3}$, Jeevitha $\mathbf{G ~ R}^{4}$, Usha Y $\mathbf{M}^{5}$ \\ ${ }^{1}$ Assistant Professor ISE Department, East West Institute of Technology, Bangalore, KA \\ 2,3,4,5B.E Students, ISE Department ,East West Institute of Technology, Bangalore, KA
}

\begin{abstract}
Individual ID numbers are broadly utilized for client verification and security. Secret word validation utilizing PINs expects clients to genuinely enter the PIN, which could be helpless against secret key splitting by means of shoulder surfing or warm following. PIN verification with hands-off look based PIN passage methods, then again, abandons no physical impressions also, hence offer a progressively secure secret phrase passage choice. Gaze based confirmation alludes to finding the eye area over consecutive picture edges, and following eye community after some time. This paper displays a continuous application for look based PIN passage, what's more, eye discovery and following for PIN recognizable proof utilizing a savvy camera.
\end{abstract}

Keywords: Face Recognition, Eye Detection, Open CV, Authentication System, HAAR Cascade.

\section{I .INTRODUCTION}

Eye trackers are the instruments that measure the visual exercises. An eye tracker records how the eyes move while a subject is finishing an assignment, for instance on a site. It catches the reflection off of the retina and the cornea of the eyes, normally alluded to as "red eye" and the flicker, separately. An eye tracker based entryway storage is an entrance control framework that permits just approved people to get to a limited zone. The framework has a PC with open CV and camera by which the secret word can be entered through it. At the point when the entered secret key equivalents with the secret word put away in the memory then the locks gets open. On the off chance that we entered an off-base secret phrase, at that point the Alert is turned on. For some having physical in ability, even basic undertaking may require assistance. Handicaps, for example, who can't communicate in and utilize gesture based communication. Assistive technology (AT) advances more prominent autonomy for individuals with handicaps by empowering them to perform errands that they were officially incapable to achieve

The present framework utilizes Open-CV apparatus for executing python codes, and HAAR Course Calculation is sent, andthe framework is made to identify the extraordinary facial qualities of the client, which is the key part in Verification Framework.

\section{LITERATURE SURVEY}

\section{Survey Paper 1}

Name of the author: Mehrube

Title:"Real-time Eye Tracking for Password Authentication"

Publication IEEE International Conference on Consumer Electronics (ICCE), 2018

Abstract: For PIN distinguishing proof, the eye place coordinates (horizontal and vertical) in the spreadsheet is first plotted on a 2Dspreadsheet. At that point the information focuses are gathered utilizing bunching. This procedure decides the looked digits, yet not the request in which they were looked. To decide the request for the entered digits, a 3D associated chart is plotted to exhibit the request for looked digits.

Limitations:

$\bullet$

$\bullet$

2.

Framework precisions less.

The soundness of the client's look will influence the precision of the identified pins.

Survey Paper 2

Name of the author: Aniwat Jubong

Title: "Smart Eye Tracking System" 
International Journal of Innovative Research in

Electrical, Electronics, Instrumentation and Control Engineering

Vol. 9, Issue 7, July 2021

DOI 10.17148/IJIREEICE.2021.9710

Publications: Research gate, 2018

Abstract: The circle Hough transform (CHT) is algorithm to detect circle, First the edge of circle will be found by using Hough gradient method after that, in each pixel on the edge of this circle another circle is created so the position that have them ost pixels accumulation from this circlecreation is center of circle.

Limitations:

- $\quad$ Web cam that connected on eye glass is huge size, it will be trouble vision of client

- $\quad$ Frame work isn't financially savvy condition.

\section{Survey Paper 3}

Name of the author: Sota Shimizu

Title:"Quantitative Analysis Of Tennis Experts 'Eye Movement Skill”

Publications: Advanced Research Institute of Science and Engineering Waseda University, 2014

Abstract: There are numerous specialists in the fields of sports, craftsmanship, and specialty. A considerable lot of these specialists can get a handle on the circumstance in a split second by their eyes, and respond on it keenly with exactness and productivity. Gadget where A Movie is shown. From the edge part of the display infra-red lights are transmitted. Cameras set in the edge some port ion of the show case measure the impression of the in fare delight from a subject's corneal.

Limitations:

-

Themeasureandexaminationoflookinformationofthesubjectisn'tdoneentirelytenniscourt.

\section{IIILITERATURE SURVEY}

\section{ARCHITECTURE}

Theframeworkdemonstratesandspeakstotheessenceofclientinaparticularregionofpicture.After that frame work will plays out a few activities of picture preparing to follow the Eye understudy. First camera module will begin to catch the pictures, and for the face location Haar course calculation is utilized. After location of legitimate face ,it will attempt to identify the eye inside the face district of intrigue. Furthermore, again Haar course calculation is utilized like as face location to distinguish eye. It will draw the rectangular box over the Eye .Presently, the fundamental objective is to identify the eye under study and characterize its middle focuses.

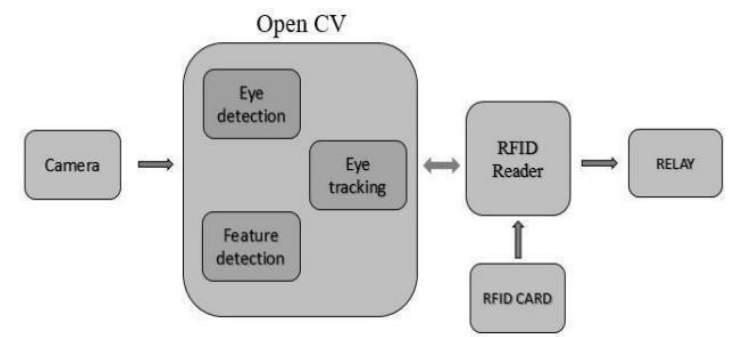

Fig1-SystemArchitecture:

Catch face pictures by means of webcam or outer USB camera,An expert HD Camera ,Faces on a picture must be distinguished

\section{METHODOLOGY}

- Obtain the midpoint.

- Draw the flat and vertical line going through the midpoint.

- Calculate the number of white pixels on each eye to the left and right of vertical line to get the gaze ratio. 
International Journal of Innovative Research in

Electrical, Electronics, Instrumentation and Control Engineering

Vol. 9, Issue 7, July 2021

DOI 10.17148/IJIREEICE.2021.9710

- Calculate the length of the flat and vertical line to get the blinking ratio.

In view of the Gaze Ratio the Left or Right Side of the console is chosen .In the wake of choosing the console the Blinking Ratio of the eye is determined. Inview of the Blinking Ratio these cretkeyis refreshed and further verification process is performed. If there should be an occurrence of substantial verification the transfer is opened else it stays shut.

\section{IV.IMPLEMENTATION}

\section{A. EYEDETECTION}

Eye detection is detection of eye region from the given image by them earns of haar cascade algorithm. Haar cascade algorithm is the machine learning object detection algorithm used to identify objects.

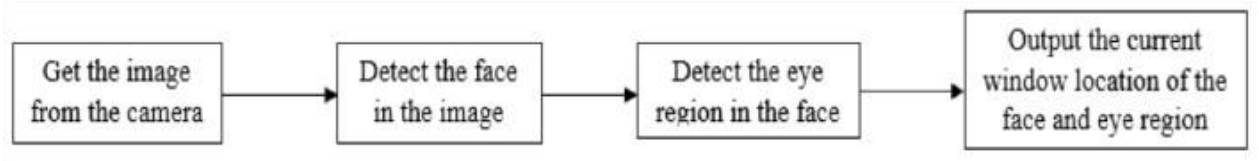

Fig2-BlockDiagram:

Haar cascade algorithm steps:

Haar feature Selection

Creating integral image

Ada boost training

Cascading Classifiers

B. FEATUREDETECTION

Similar to eye detection, feature detection is detecting official key structure and locating the detected facial structure with specific $(\mathrm{x}, \mathrm{y})$ co-ordinates values. Then the co-ordinate values of the left and right eyes are considered and the polygon is drawn over the eye region.

Facial Landmark detector algorithm is used to achieve the above process.

\section{Facial Landmark Algorithm-}

* Input the window location where the face and eye region is found.

* Detect the key facial structures in the image.

* Locate the key facial structures with specific (x, y) co-ordinates.

o Startwith1 for first(x, y)co-ordinate.

o Endwith68forthelast(x, y)co-ordinate.

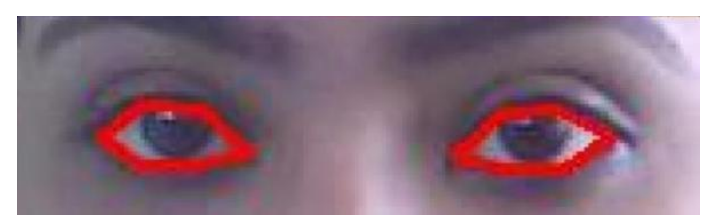

Fig3-Faciallandmarkpoints:

\section{C.EYETRACKING}

Here the eye movement is continuously tracked to obtain the Gaze Ratio and based on the gaze ratio the respective keyboard will be displayed. Then the eye blinking ratio will be calculated to up date the respective letter as the password.

Algorithm: To calculate the gaze ratio,

1. Input the pixel values of the eye region. 


\section{International Journal of Innovative Research in \\ Vol. 9, Issue 7, July 2021 \\ DOI 10.17148/IJIREEICE.2021.9710} Electrical, Electronics, Instrumentation and Control Engineering

2. Get only the eye region.

3. Divide each eye region in to left and right part.

4. Convert the eye image into grey scale.

5. Get the number of white pixels on both side i.e., on the left side and right side of each eye.

6. Calculate the Gaze Ratio:

i. G1; Gaze Ratio (left eye) $=$ No. of white pixels on right side by No. of white pixels on the left side.

ii. $\quad$ G2; Gaze Ratio (right eye) = No. of white pixels on left side by No. of white pixels on the right side.

iii.

7. If (Gaze Ratio <=0.9)then select the right keyboard Else

Select the left keyboard

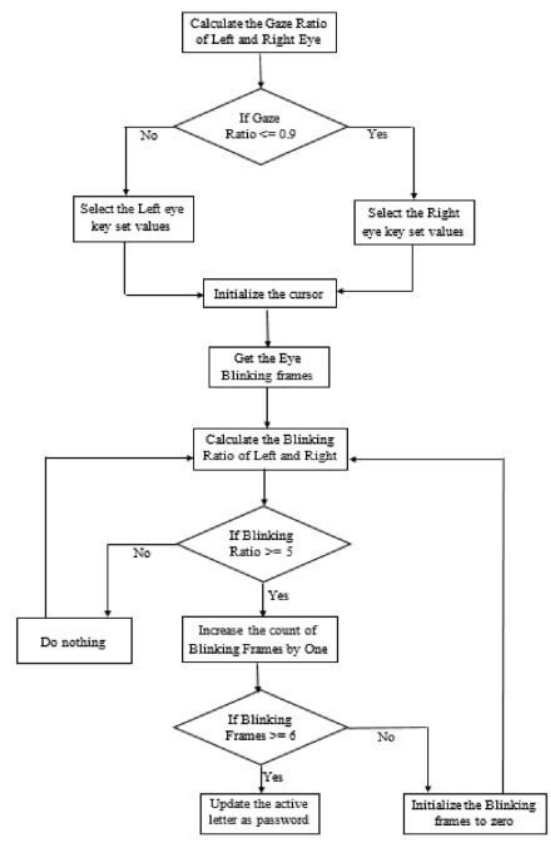

Fig 4-DataFlow Diagram:

\section{V.REQUIREMENTS}

\section{A.FUNCTIONALREQUIREMENTS}

1. Face and Eye identification:

Framework will detect and differentiate between the face and the eye of an individual from the picture caught by the camera.

\section{Feature extraction:}

Frame work will extricate the eye district then the eye understudy area and this locale is stamped and the eye student focus will be found.

\section{Eye tracking:}

Framework will follow the eye student development and imprint the eye understudy focus on arrange frame work.

\section{PIN identification:}

Framework

will

recognize

the

PIN

dependent

on

the 
International Journal of Innovative Research in

Electrical, Electronics, Instrumentation and Control Engineering

Vol. 9, Issue 7, July 2021

DOI 10.17148/IJIREEICE.2021.9710

pointthatissituatedonthearrangeframeworkandtherefreshedsecretphrasewillbesentforadditionalvalidationprocedure.

5. Authentication:

When the frame work gets these cretphrase the confirmation procedure will be done to check whether the secret phrase entered is substantial or not. With the go althattheun approved client can't get to there cord.

6. Authorization:

In the event that the entered secret word is substantial, at that point the frame work permits to open withthegoalthatclientcanadditionallydohisexchangeselselockerremainsbolted.

\section{B.SOFTWAREREQUIREMENTS}

- Windows 10/ Linux is the operation system we are using to achieve the project goals.

- The coding Language is Python.

- TheIntegratedDevelopmentEnvironmentisacodingtoolwhichallowsyoutowrite,test,anddebugthecode in an easier way.

- The Integrated Development Environment is anOpen source computer vision.

\section{C.HARDWAREREQUIREMENTS}

- $\quad$ Pentium IV $2.4 \mathrm{GHz}$ is the Intel processor, it has a viable clock speed that now exceeds2 $\mathrm{GHz}$.

- HD-3000 Webcam is a camera that feeds orstreams nimageorvideo in real time.

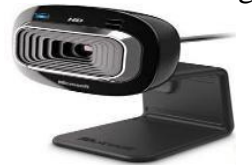

Fig2:Camera

- $\quad$ Logitech wireless mouse is a reliable due to long-range, advanced connectivity.

- $\quad$ A hard disk is a magnetic storage device for digital data ,it can store large amounts of data and the hard disk is of $40 \mathrm{~Gb}$.

- $\quad$ A Video Graphic Array (VGA) is a cable used to connect an analog PC monitor to PC/Laptop.

\section{VI.SYSTEMOUTCOMES}

The fundamental working standard of the undertaking is that, the conceivable method for secret phrase verification by utilizing continuous eye following. A shrewd camera based eye-following framework has been fused into another application for look based PIN ID.

\section{A. Capture Face:}

First camera module will begin to catch the pictures, and for the face location Haar course calculation is utilized. After location of appropriate face, it will attempt to identify the eye inside the face locale of intrigue.

\section{B. Face Detection:}

Face Detection is where the picture, given as an info (picture) is looked to discover any face, in the wake of finding the face the picture handling tidiesup the facial picture for simpler acknowledgment of the face.

\section{Face Recognition using HAAR Cascade:}

After the fulfillment of identifying and handling the face, Haar course calculation is utilized like as face recognition to distinguish eye.

It will draw the rectangular box over the Eye. Presently, the fundamental objective is to identify the eye student and characterize its middle focuses.

\section{VII.CONCLUSION}

In our present research, we talked about conceivable method for secret word verification by utilizing continuous eye following. A shrewd camera based eye-following framework has been fused into another application for look based PIN recognizable proof. 


\section{International Journal of Innovative Research in \\ Vol. 9, Issue 7, July 2021 \\ DOI 10.17148/IJIREEICE.2021.9710} Electrical, Electronics, Instrumentation and Control Engineering

\section{REFERENCES:}

[1] Sarangi,P.,Grassi,V.,Kumar,V.,Okamoto,J.:’Integrating Human Input autonomous behavioursonanIntelligentWheelchairPlatform”, JournalofIEEEIntelligent System,22,2, 33-41,[2007].

[2] MattBailey,ET.A1,“DevelopmentofVisionBasedNavigationforaRoboticWheelchair",inProceedingsof2007 IEEE 10th International conference on rehabilitationrobotics.

[3] Shafi.M,Chung.P.W.H:”AHybridMethodforEyesDetectioninFacialImages",InternationalJournalofElectrical,Computer,andSystemsEngi neering,231-236,[2009].

[4] Automationofwheelchair usingultrasonicandbodykinematics, Preethika Britto, Indumathi. J, Sudesh Sivarasu,LazarMathew,CSIOChandigarh,INDIA, 19-20March2010.

[5] Poonam S. Gajwani \& Sharda A. Chhabria, "Eye MotionTracking for Wheelchair Control", International journal ofinformation technology and knowledge management, Dec2010.EyeControlledWheelChairUsingEOG,AlexDev,HorizonCChackoandRoshanVarghese,InternationalConference on Computing and Control Engineering (ICCCE2012),12\&13April,2012. 